\title{
Appearance-consistent Video Object Segmentation Based on a Multinomial Event Model
}

\author{
YADANG CHEN, Nanjing University of Information Science and Technology, China \\ CHUANYAN HAO, Nanjing University of Posts and Telecommunications, China \\ ALEX X. LIU, Michigan State University, USA and Nanjing University, China \\ ENHUA WU, University of Chinese Academy of Sciences, China
}

\begin{abstract}
In this study, we propose an effective and efficient algorithm for unconstrained video object segmentation, which is achieved in a Markov random field (MRF). In the MRF graph, each node is modeled as a superpixel and labeled as either foreground or background during the segmentation process. The unary potential is computed for each node by learning a transductive SVM classifier under supervision by a few labeled frames. The pairwise potential is used for the spatial-temporal smoothness. In addition, a high-order potential based on the multinomial event model is employed to enhance the appearance consistency throughout the frames. To minimize this intractable feature, we also introduce a more efficient technique that simply extends the original MRF structure. The proposed approach was evaluated in experiments with different measures and the results based on a benchmark demonstrated its effectiveness compared with other state-of-the-art algorithms.
\end{abstract}

CCS Concepts: • Computing methodologies $\rightarrow$ Video segmentation; Object detection; Supervised learning;

Additional Key Words and Phrases: Multinomial event model, appearance consistency, Markov random field

ACM Reference format:

Yadang Chen, Chuanyan Hao, Alex X. Liu, and Enhua Wu. 2019. Appearance-consistent Video Object Segmentation Based on a Multinomial Event Model. ACM Trans. Multimedia Comput. Commun. Appl. 15, 2, Article 40 (May 2019), 15 pages.

https://doi.org/10.1145/3321507

This work is partially supported by the National Natural Science Foundation of China (Grants No. 61802197, No. 61702278, No. 61872082, No. 61472184, No. 61703212, and No. 61602252), the National Science Foundation (Grant No. CNS-1837146), the Natural Science Foundation of Jiangsu Province of China (Grants No. BK20160964, No. BK20160902, No. BK20160971, and No. BK20160967), the Project through the Priority Academic Program Development (PAPD) of Jiangsu Higher Education Institutions, and the Jiangsu Innovation and Entrepreneurship (Shuangchuang) Program.

Authors' addresses: Y. Chen, School of Computer and Software, Nanjing University of Information Science and Technology, Nanjing, 210044, China; email: cyd4511632@gmail.com; C. Hao, School of Education Science and Technology, Nanjing University of Posts and Telecommunications, Nanjing, 210023, China; email: hcy@njupt.edu.cn; A. X. Liu, Department of Computer Science and Engineering, Michigan State University, East Lansing, MI, 48824-1266, USA; State Key Laboratory for Novel Software Technology, Nanjing University, Nanjing, 210044, China; email: alexliu@cse.msu.edu; E. Wu, State Key Laboratory of Computer Science, Institute of Software, University of Chinese Academy of Sciences, Beijing, 100864, China; email: ehwu@umac.mo.

Permission to make digital or hard copies of all or part of this work for personal or classroom use is granted without fee provided that copies are not made or distributed for profit or commercial advantage and that copies bear this notice and the full citation on the first page. Copyrights for components of this work owned by others than ACM must be honored. Abstracting with credit is permitted. To copy otherwise, or republish, to post on servers or to redistribute to lists, requires prior specific permission and/or a fee. Request permissions from permissions@acm.org.

(C) 2019 Association for Computing Machinery.

1551-6857/2019/05-ART40 \$15.00

https://doi.org/10.1145/3321507

ACM Trans. Multimedia Comput. Commun. Appl., Vol. 15, No. 2, Article 40. Publication date: May 2019. 


\section{INTRODUCTION}

The video object segmentation task involves separating foreground objects from the background in a video sequence. This binary segmentation problem has been addressed using a number of different methods [1, 15, 27, 29]. These approaches can be divided into unsupervised methods $[13,16,37,40]$, where the video is the only input, and supervised methods [2, 3, 26, 32], which require that a user provides the initial labels. Defining what constitutes an "interesting object" is often specific to the application, and the same video could have multiple valid solutions. Thus, we consider the supervised approach in the present study.

In many of the existing video object segmentation approaches, graph-based methods provide a promising framework for propagating foreground regions in video [3, 21, 32, 40]. In general, these methods decompose each frame into spatio-temporal nodes, thereby casting the segmentation problem as a labeling problem in a Markov random field (MRF). Most of these methods [2, 3, 21, 32] search for the foreground-background label assignment that minimizes both the unary potential with the supplied labeled frames as well as the smoothness potential in space and time. Despite encouraging results, these methods are affected by the following important technical challenge. Reliable foreground segmentation in video requires the capture of long-range connections as an object moves and its shape changes over time, but these methods restrict the graph connectivity to local cliques that can be noisy due to many factors, e.g., irregularly shaped objects and imperfect frame-to-frame optical flow.

In our proposed method, an additional high-order potential [10] is employed for video object segmentation. In particular, the entire video is treated as a large collection of visual words. We then compute the potential for each node using the multinomial event model, which is generally employed for text classification [22], where the nodes that have similar visual features are specified as soft preferences for assignment with the same label (foreground or background). This global clique is the key to our approach and it ensures long-term appearance consistency during segmentation. Inspired by previous studies [40], we also introduce a more efficient method based on simply extending the original MRF structure for optimizing the high-order potential.

In summary, the contributions of this work are the following. First, we propose a fast and accurate video object segmentation method, where the initial object mask is given in a few frames of an input video. Second, a mathematical sound method based on the MRF model is employed to address the video object segmentation problem. Third, a high-order potential based on the multinomial event model is employed to enhance the appearance consistency throughout the frames, which can be solved efficiently by the adoption of additional auxiliary nodes and edges. The proposed method was evaluated qualitatively and quantitatively in experiments, and the results demonstrated its effectiveness.

\section{RELATED WORK}

Unsupervised Segmentation. Unsupervised segmentation aims to extract the object from the whole video without any manual annotation. HVS [7] and LIBSVX [38] generate an oversegmentation of the video as spatio-temporal segments with uniform motion and appearance, which are important for early stage video preprocessing, but they do not directly solve the problem of video object segmentation. In FST [25] and POLP [5], the oversegmentation method is extended by clustering segments into foreground and background segments according to the distance measured between them in a high-level feature space. Other recent state-of-the-art methods, e.g., SAG [35], TS [37], and ARP [16], generate a considerable number of object segments and rank them for each frame, and thus the video object segmentation task is transformed into a selection problem. These automatic video segmentation methods certainly have advantages, but they only work with 
constrained videos, where the salience of a "desired object" is quite different from the background. For general purposes, we must instead focus on the user-assisted approach.

Trajectory Tracking. In general, supervised methods require that the user manually annotates a few frames, before propagating these annotations automatically to the remaining video frames, where they focus on the label inference problem. The approach known as point tracking, e.g., as applied in CUT [14] and LTA [23], attempts to match each pixel $x$ in the target frame $I_{t g}$ with a pixel $y$ in the source frame $I_{s r}$ by computing a dense correspondence. The label inference can then be set as $L_{t g}(x)=L_{s r}(y)$. The dense correspondence can be clustered based on the sparse trajectories or feature points, but it is unreliable when the matching contains rotations, occlusions, large movements, and other features. Furthermore, the method proposed by References [9, 33] exploits occlusion detection to discard unreliable trajectories, while that developed by References $[3,24]$ separates the motion estimates for the foreground and background from each other to improve the tracking. However, the occlusion problem remains unsolved.

Graph-based methods. Another alternative approach is based on probability graphs [10, 21, 28, 32], where various classifiers used for segmenting an unlabeled frame are trained based on labeled frames. It has been shown that these well-trained classifiers are effective for handling occlusions. In particular, JOTS [36] builds a graph over the neighboring frames that connect superpixels and (generic) object parts to solve the video labeling task. OFL [32] builds a graph over pixels and superpixels, before using ConvNet-based appearance terms, and interleaving labeling with optical flow estimation. Instead of using superpixels or proposals, BVS [21] formulates a fully connected pixellevel graph between frames and efficiently infers the labels over the vertices of a spatio-temporal bilateral grid. However, short-range connections that only cross neighboring frames cannot capture the long-range temporal changes in an object.

To model long-range temporal connections, NLC [4] uses a random walk transition matrix to diffuse the object similarity between video frames. In addition, TVS [34] computes the similarity in a temporal tree structure. Moreover, FCP [28] builds a global graph structure over the proposed object segments and then infers a consistent segmentation. A limitation of these methods is that they employ an iterative approach for optimization, which is readily trapped by local optima and it is time consuming, especially for video data. By contrast, we introduce a more efficient method without iteration.

End-to-end CNN Segmentation. Recently, CNN-based methods [11, 12, 26] have been developed as novel approaches for video object segmentation by combining offline and online training processes using static images. Significant performance improvements have been achieved using these end-to-end approaches, where the networks need to be pre-trained with a large-scale annotated data set. In the present study, we still focus on the traditional graph-based method.

\section{PROPOSED APPROACH}

Given a video sequence $V$, our objective is to take the foreground object segmentation drawn on a few frames $S$ and accurately propagate it to the remainder of the frames $\bar{S}$, where $V=S \cup \bar{S}$. Similar to other graph-based approaches, we transform the segmentation problem into a labeling problem in a MRF. The general framework of the proposed approach is illustrated in Figure 1. First, we over-segment each video frame into a set of superpixels. We then construct the segmentation graph using all of the superpixels and a number of auxiliary nodes. Finally, the segmentation result can be solved by any MRF optimization algorithm. Furthermore, we denote the label of superpixel $x$ as $l(x)$, where $x \in V$ and $l(x) \in\{+1,-1\}$. Thus, our method can be formulated as

$$
l^{*}(X)=\arg \min _{l(X)} E(X)=\arg \min _{l(X)} E_{u}(X)+\alpha E_{p}(X)+\beta E_{h}(X),
$$



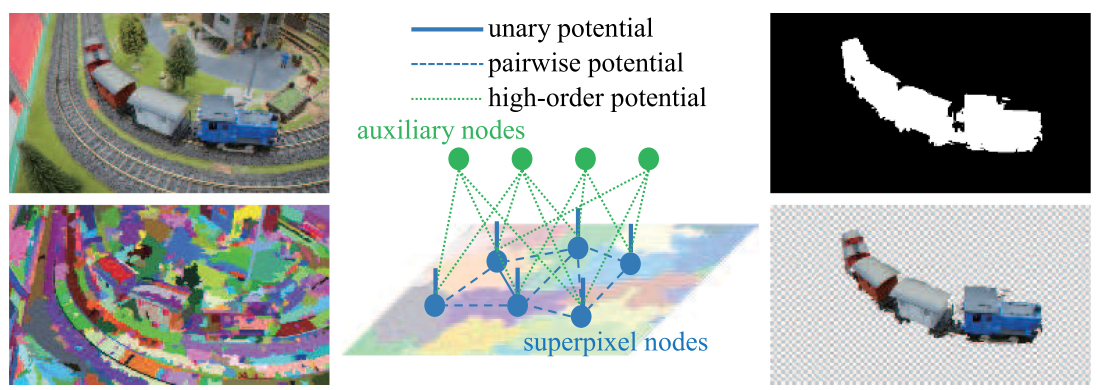

Fig. 1. Overview of the proposed method. From top to bottom and left to right: video frame, generating superpixels, graph construction, binary mask, and final segmentation result.

where $X=\{x \mid x \in \bar{S}\}, E_{u}, E_{p}$, and $E_{h}$ are the unary potential, pairwise potential, and high-order potential, respectively, and $\alpha$ and $\beta$ are the weights for the linear combination. Each part of this method is described in detail in the following.

\subsection{Adaptive Over-segmentation}

In our method, we model each node as a superpixel, because using pixels incurs a high computational cost and can also be more sensitive to noise. Large superpixels are likely to contain pixels from the foreground and background, because the object boundary might not be clear. Small superpixels are less informative but they can be used to obtain more accurate estimates for segmentation. Thus, we employ an adaptive method based on the HVS algorithm [7]. HVS begins with a fine over-segmentation and the regions are then merged to form larger super-regions during the next iteration. This algorithm saves the hierarchical results as a tree-structure, which allows the desired segmentation to be selected at any desired level of granularity. Before the next iteration, we compute two more values for each $\{x \mid x \in S\}$ :

$$
\left\{\begin{array}{l}
r_{a}(x)=\frac{x \cap G}{G}, x \in S, \\
r_{b}(x)=\frac{x \backslash(x \cap G)}{G}, x \in S,
\end{array}\right.
$$

where $G$ denotes the ground truth of object. If $\exists x$ meets both $r_{a}(x)>\sigma_{r}$ and $r_{b}(x)>\sigma_{r}$ simultaneously, then we stop iterating. The result saved in the previous layer is considered the most appropriate. $\sigma_{r}$ is a threshold to control the size of superpixels. A larger $\sigma_{r}$ usually leads to larger superpixels but also a higher likelihood of missed segmentation boundaries. We empirically set $\sigma_{r}=0.05$ in the experiments. Figure 2 shows that our method is adaptive with different videos.

\subsection{Unary Potential}

We use the bag-of-words approach [20] to describe the irregularly shaped superpixels. Given the entire video, we quantize the average RGB colors of all the superpixels into 100 visual words and associate each pixel with the nearest word. We then construct a histogram of the words for the pixels within $x$ and normalize it into the feature vector $f(x)$.

Subsequently, all of the features are evaluated by learning a transductive SVM classifier, as illustrated in Figure 3. For a good learning, only "easy" samples are fed. In our experiments, the superpixels $\{x \mid x \in S\}$ with $0.8 \leqslant r_{a}(x) \leqslant 1.0$ are selected as positive samples, whereas those within $[0,0.1]$ are selected as negative samples. Let $\{f(1), \ldots, f(x), \ldots, f(n)\}$ denote the features extracted from the entire video, including both the labeled and the unlabeled superpixels, where the labels are denoted as $\{l(1), \ldots, l(x), \ldots, l(n)\} \in\{-1,+1\}^{n}$. We then assume that the first $m$ features 

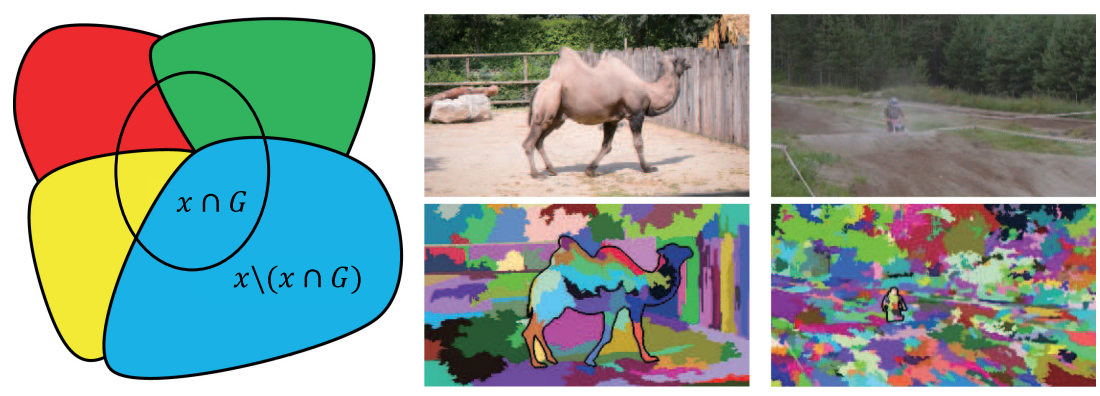

Fig. 2. Proposed adaptive over-segmentation method. Left: the colored regions denote different superpixels and the circle in the middle denotes the ground truth of object. Taking the blue superpixel, for example, $x \cap G$ and $x \backslash(x \cap G)$ are the respective regions as shown. Right: our proposed method generates large superpixels (ninth iteration) for the "camel" data set and small superpixels for the "motocross" data set (third iteration).

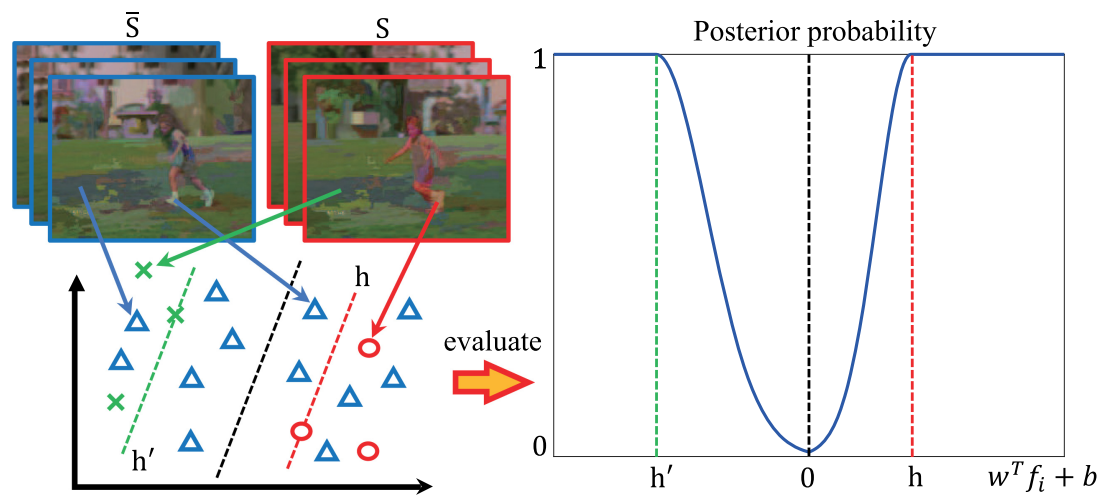

Fig. 3. Left: Transductive learning where the positive (red) and negative (green) examples are generated from $S$, whereas the other unlabeled examples (blue) are generated from $\bar{S}$. Right: Illustration of the proposed piecewise function, which makes the confidence decrease when the example is close to the separating hyperplane, whereas it increases otherwise.

have been labeled as positive or negative, and the problem can be represented by

$$
\begin{array}{r}
\min _{w, b,\{l(m+1), \ldots, l(n)\}} \frac{1}{2}\|w\|^{2}+C \sum_{x=1}^{n} \xi_{x}, \\
\text { s.t. }\left\{\begin{array}{l}
l(x)\left(w^{T} f(x)+b\right) \geq 1-\xi_{x}, x=1, \ldots, n \\
\xi_{x} \geq 0, x=1, \ldots, n .
\end{array}\right.
\end{array}
$$

To obtain an efficient solution, we apply the convex relaxation formulation [39] by changing the non-convex optimization problem into its dual problem. More details of this solution were given by Reference [39]. By using the learned parameters $w$ and $b$, we also evaluate the posterior probability for each assignment by

$$
p(x)=\left\{\begin{array}{l}
\exp \left(-\frac{\|h(x)-h\|^{2}}{2\left(\frac{h}{3}\right)^{2}}\right), h(x)<h, \\
1, h(x) \geq h,
\end{array}\right.
$$

where

$$
h(x)=l(x)\left(w^{T} f(x)+b\right)
$$


and

$$
h=\max \left(1, \underset{l(y)=l(x)}{y \in\{1, \ldots, m\}} l(y)\left(w^{T} f(y)+b\right)\right) .
$$

An illustration of the piecewise function given above is shown in Figure 3, which shows that the confidence decreases when the example is close to the separating hyperplane, whereas it increases otherwise. In particular, the examples more distant than the nearest labeled examples (positive and negative, respectively) are considered as those with full confidence.

Thus, the unary potential in Equation (1) is defined as

$$
E_{u}(X)=\sum_{x}\left[l(x) \neq l^{\prime}(x)\right] \cdot p(x)
$$

where $[*]=1$ when $*$ is true; otherwise, $[*]=0 . l^{\prime}(x)$ is the label assigned by the transductive SVM classifier.

\subsection{Pairwise Potential}

Similar to other methods, the pairwise potential is mainly used for the spatial-temporal smoothness in the proposed approach. Two superpixels are spatially connected if they share one edge and two superpixels are temporally connected if they are in one supervoxel pre-segmented by HVS [7] mentioned in Section 3.1. We denote the collections of all the spatial pairs and all the temporal pairs as $\varepsilon_{s}$ and $\varepsilon_{t}$, respectively. Thus, the pairwise potential is defined as

$$
E_{p}(X)=\sum_{\left(x, x^{\prime}\right) \in \varepsilon_{s} \cup \varepsilon_{t}} E_{p}\left(x, x^{\prime}\right) .
$$

We measure the similarity between a spatial pair based on both the color features,

$$
E_{p}\left(x, x^{\prime}\right)=\left[l(x) \neq l\left(x^{\prime}\right)\right] \cdot \exp \left(-\frac{\left\|f(x)-f\left(x^{\prime}\right)\right\|^{2}}{\sigma_{c}^{2}}\right),
$$

where $\sigma_{c}$ is the scaling parameters for visual dissimilarity.

\subsection{High-order Potential}

Finally, we define the last potential in Equation (1). The proposed method builds on the multinomial event model, which is generally used for text classification [22], so we start with a brief overview of the model. Let $x$ denote the text, $l(x) \in\{+1,-1\}$ denote its label, $n_{x}$ denote the length of $x$, and $x_{i}$ denote the identity of the $i$ th word in $x$. Thus, $x_{i}$ is now an integer that takes values in $\{1, \ldots, k, \ldots,|D|\}$, where $|D|$ is the size of the dictionary. Next, we construct a histogram $H$ with dimensionality $|D|$. Furthermore, $H_{k}^{+1}$ and $H_{k}^{-1}$ are used to denote the number of words assigned to the $k$ th bin for the " +1 " and " -1 " texts, respectively, i.e., $H_{k}^{+1}=\sum_{x} \sum_{i=1}^{n_{x}}[l(x)=1]\left[x_{i}=k\right]$, and $H_{k}^{-1}=\sum_{x} \sum_{i=1}^{n_{x}}[l(x)=-1]\left[x_{i}=k\right] . H_{k}$ is used to denote the total number, i.e., $H_{k}=H_{k}^{+1}+H_{k}^{-1}$. Thus, the " +1 " and " -1 " probabilities of the $k$ th histogram bin are computed by $p_{k}^{+1}=H_{k}^{+1} / H_{k}$ and $p_{k}^{-1}=H_{k}^{-1} / H_{k}$, respectively. According to the naive Bayes assumption, the " +1 " and " -1 " probabilities of text $x$ can be approximated by $p(+1 \mid x)=\prod_{i=1}^{n_{x}} p_{x_{i}}^{+1}$ and $p(-1 \mid x)=\prod_{i=1}^{n_{x}} p_{x_{i}}^{-1}$, respectively.

We treat each superpixel $x$ as a text. A naive method for describing the "words" in $x$ is to use the color histogram $f(x)$. However, the raw color features may not be sufficiently robust to accurately capture the viewpoint (please refer to Section 4.2). Hence, we propose to use the more distinctive SIFT feature. In particular, the SIFT key points are extracted from the entire video, and we then cluster all of these $128 d$ features into $|D|$ words that form the visual dictionary. Now, each 


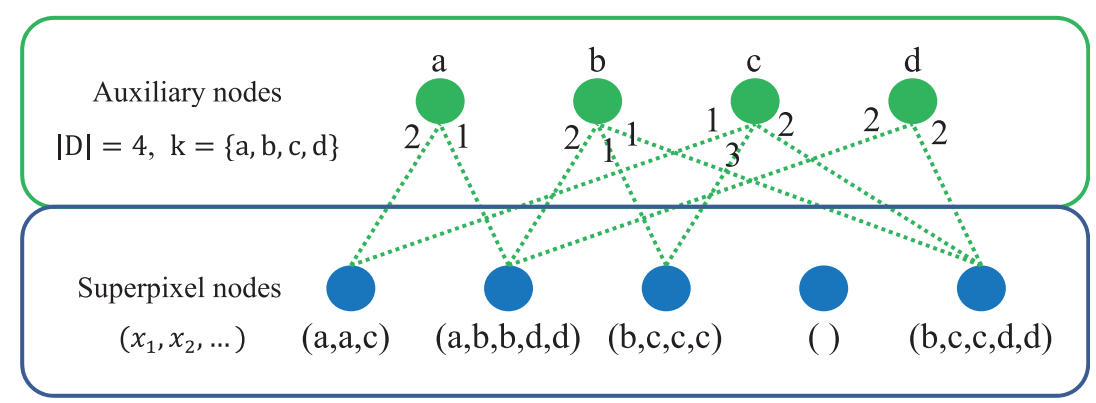

Fig. 4. Example illustrating how the auxiliary nodes and edges are added to the original MRF graph. In this example, the size of the dictionary is only 4 . The weight of the connection between the superpixel node $x$ and the auxiliary node $k$ is equal to the number of words where $x_{i}=k$ in $x$. The connections between the superpixel nodes are omitted for simplicity.

superpixel $x$ contains $n_{x}$ words. Finally, the potential is formulated as

$$
E_{h}(X)=\sum_{x}-\ln p(l(x) \mid x)=\sum_{x}-\ln \prod_{i=1}^{n_{x}} p_{x_{i}}^{l(x)},
$$

where $p_{x_{i}}^{l(x)}=H_{x_{i}}^{l(x)} / H_{x_{i}}$ builds a global clique for each $x$, which is crucial for our method, because it ensures long-term appearance consistency during segmentation.

To minimize the energy $E_{h}(X)$, we could use the $\alpha$-expansion algorithm [17] implemented in a complicated iterative process. However, based on the method proposed by Reference [40], we introduce a more efficient approach to solving this problem. Let $v_{x}^{k}$ denote the number of words where $x_{i}=k$ in $x$ and $p(l(x) \mid x)$ can be redefined as $\prod_{k=1}^{|D|}\left(p_{k}^{l(x)}\right)^{v_{x}^{k}}$. Thus, Equation (10) can be rewritten as

$$
\begin{aligned}
E_{h}(X) & =\sum_{x}-\ln p(l(x) \mid x)=\sum_{x}-\ln \prod_{k=1}^{|D|}\left(p_{k}^{l(x)}\right)^{v_{x}^{k}} \\
& =-\sum_{x} \sum_{k=1}^{|D|} v_{x}^{k} \ln p_{k}^{l(x)}=-\sum_{k=1}^{|D|} \ln p_{k}^{l(x)} \sum_{x} v_{x}^{k} \\
& =-\sum_{k=1}^{|D|} \ln p_{k}^{l(x)} H_{k}=-\sum_{k=1}^{|D|}\left(H_{k}^{+1} \ln p_{k}^{+1}+H_{k}^{-1} \ln p_{k}^{-1}\right) \\
& =-\sum_{k=1}^{|D|}\left(H_{k}^{+1} \ln \frac{H_{k}^{+1}}{H_{k}}+H_{k}^{-1} \ln \frac{H_{k}^{-1}}{H_{k}}\right) .
\end{aligned}
$$

$H_{k}^{+1}+H_{k}^{-1}$ is a fixed number, so the symmetric function $\left(H_{k}^{+1} \ln H_{k}^{+1} / H_{k}+H_{k}^{-1} \ln H_{k}^{-1} / H_{k}\right)$ has a minimum value when $H_{k}^{+1}=H_{k}^{-1}$. Thus, $E_{h}(X)$ can be approximated as

$$
E_{h}(X) \approx-\sum_{k=1}^{|D|}\left|H_{k}^{+1}-H_{k}^{-1}\right| \approx \sum_{k=1}^{|D|} \min \left\{H_{k}^{+1}, H_{k}^{-1}\right\} .
$$

Compared with Equation (10), the optimization of Equation (12) can be solved more easily by simply adding some auxiliary nodes and edges to the original MRF graph, as illustrated in Figure 4. In particular, we add $|D|$ auxiliary nodes to the graph, where each auxiliary node denotes a bin of the histogram $H$. Each superpixel $x$ in the graph is then connected with its assigned bins. The 
unary potentials of all the auxiliary nodes are set to 0 , and the pairwise potential of the edge connecting $x$ and $k$ th auxiliary node is set to $[l(x) \neq l(k)] \cdot v_{x}^{k}$.

At this point, the segmentation graph has been obtained, which can be solved by any MRF optimization algorithm. By using the added auxiliary nodes and edges, the superpixels with similar visual features throughout the video have soft preferences that need to be assigned the same label.

\section{EXPERIMENTS}

\subsection{Data Sets and Parameters}

The well-known SegTrack data set [31] has been used frequently to assess the performance of video object segmentation with conventional methods, where it comprises six videos. SegTrack.v2 [19] extends the SegTrack data set by providing eight challenging video sequences. Recently, Perazzi et al. [27] published a new benchmark called the DAVIS data set, which comprises a total of 50 highresolution (480P) sequences alternating with a wide range of object segmentation challenges, such as occlusion, fast motion, and appearance changes. Thus, we evaluated the proposed algorithm based on these three data sets and their available pixel-wise ground-truth maps.

The unary and pairwise terms have been explored in most of the MRF-based approaches, so we empirically set $\alpha=0.3$ in Equation (1), $\sigma_{c}=0.1$ in Equation (9), to prevent each of them from being dominated by the others. We also set the size of the SIFT dictionary as $|D|=100$. These parameters were fixed in all of the experiments. In terms of the weight of the high-order potential, we set $\beta=1 \operatorname{avg}\left(n_{x}\right)$ to maintain a balance between the items in Equation (1), where $\operatorname{avg}\left(n_{x}\right)$ computes the average length of all the superpixels. Moreover, we used five labeled frames for each video to ensure that the transductive learning process had sufficient samples to train a valid model.

\subsection{Ablation Study}

To evaluate the proposed method, ablation analysis was conducted for the components in Equation (1). In particular, we compared our method with three baseline approaches. The first segmentation was achieved only with the unary item, i.e., $E(X)=E_{u}(X)$, which was designed to evaluate the transductive learning process. The second was designed without the high-order item, i.e., the energy became $E(X)=E_{u}(X)+\alpha E_{p}(X)$. The last was designed to use a naive $E_{h}(X)$, where the "words" in superpixel $x$ were described using $f(x)$. In this approach, the $k$ th auxiliary node denotes the $k$ th bin of the color histogram and $x$ is connected with its assigned auxiliary nodes according to the colors of the pixels contained.

Figure 5(a) shows the average intersection-over-union (IoU) scores based on different benchmarks and Figure 6 gives some examples. As expected, our method always performed better than the other baseline approaches. However, the result was usually too crude with only the unary term because of the inevitable misclassifications. The results could be improved by the local cliques $E_{p}(X)$, but they were still noisy due to the misaligned motion matching. With the naive $E_{h}(X)$, we found that the results were better due to the proposed global constraint. However, the algorithm still confused the foreground and background when similar colors were present between them, because the foreground and background superpixels were connected to the same color bin with high weights, thereby leading to the same label. In contrast to these baseline approaches, our method employs the more distinctive SIFT feature instead of the raw colors and it ensures long-term appearance consistency throughout all of the frames to achieve higher accuracy, as illustrated by the results.

Moreover, besides the bag-of-words approach [20] for describing the superpixels, we also test the performance on several CNN-based feature descriptors. Specifically, we fill the boundary box of the irregularly shaped superpixel with its average color [6] and encode the rescaled patch by 
(a)

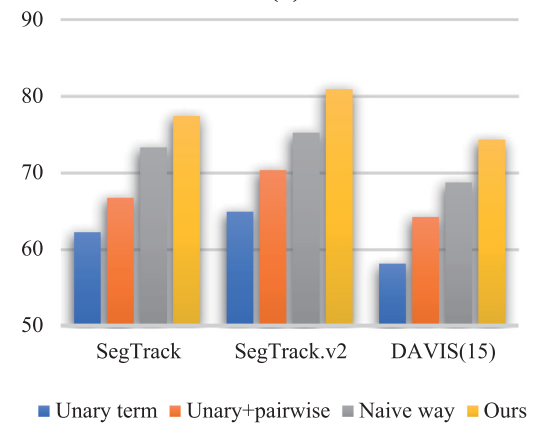

(b)

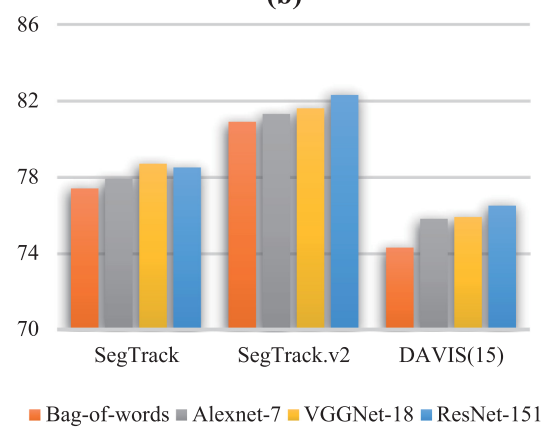

Fig. 5. (a) Average intersection-over-union (IoU) scores based on different benchmarks. (b) The average loU scores by different feature descriptors. Note that DAVIS(15) is a subset of the DAVIS data set that includes 15 randomly selected videos to reduce the workload.

different networks that are pre-trained on ImageNet classification, to acquire high-level semantic features. The 7th layer of AlexNet [18], the 18th layer of VGGNet [30], and the 151th layer of ResNet [8] are selected as the outputs for this evaluation, respectively. From the result shown in Figure 5(b), we find that the improvement is not obvious, especially for SegTrack and SegTrack.v2. There are two reasons: First, the filling operation causes feature distortion, which is explained in Reference [6]. Second, the CNN-based feature descriptor cannot do its best without the online fine-tuning step for a special application. Thus, we prefer using the bag-of-words method in this article for high efficiency and low complexity.

\subsection{Comparison with Peer Methods}

We compare the performance of the proposed method with other video segmentation methods, including both unsupervised and supervised methods, based on different benchmarks. Since most of the supervised methods are designed to use only one labeled frame (e.g., the first frame) for initialization, we give both IoU scores with one labeled frame "ours(1)" and five labeled frames "ours(5)" individually for the proposed method.

SegTrack: A primary object in each video maintains a similar size throughout all of the frames, so the average pixel error (APE), i.e., the average number of mislabeled pixels per frame, is used as the performance metric in most of the conventional approaches. Table 1 compares our results and those reported previously. Moreover, simply averaging the APE over all videos is unreasonable, because it is sensitive to the object size. Therefore, we determined the additional APE rankings for these methods and also computed the average rank over the first five sequences, because many studies did not report the performance for "penguin." These results demonstrate that "ours(5)" achieved the best rank on average. Even "ours(1)" achieves a good level in comparison to other methods, in particular being the second highest among the supervised methods, where TVS[34] approach requires more than one labeled frame for initialization.

SegTrack.v2: Table 2 presents the IoU scores based on SegTrack.v2 [19] compared with some recent approaches that reported their results in terms of the same metric. The average score was also computed over all the listed sequences for each method. According to these results, our method failed to perform better than the others for "soldier" and "monkey" due to the coarse oversegmentation and indistinctive features of the object. More details of these errors are provided in Section 4.5. However, it remained competitive with these methods, where OFL [32] benefited considerably from complicated iterative optimization, which requires more computation costs. 

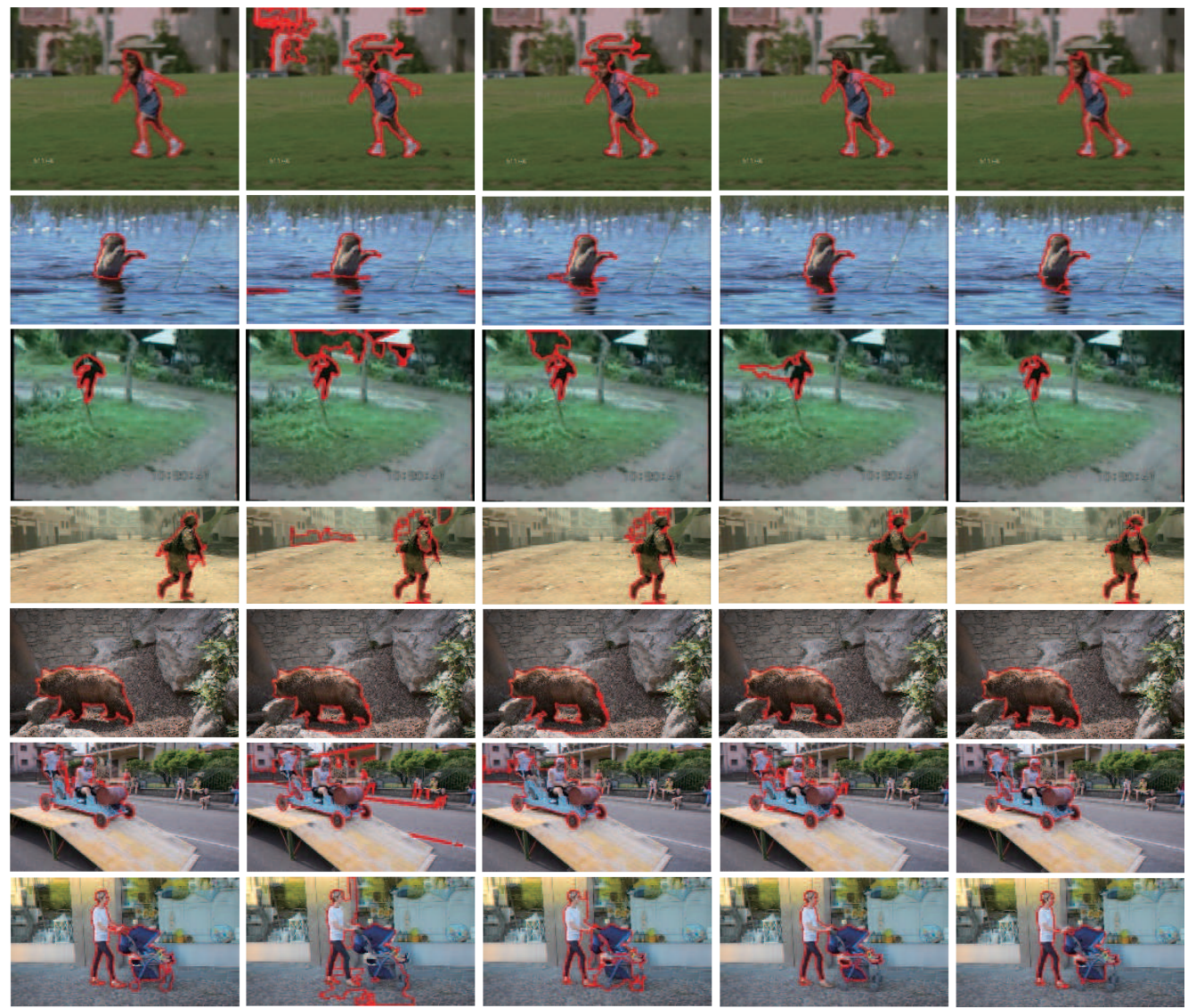

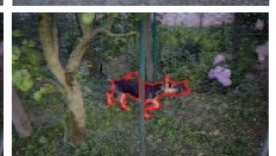

Without $E_{h}(X)$

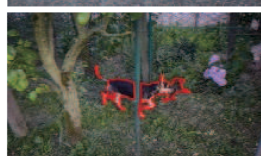

Naive $E_{h}(X)$

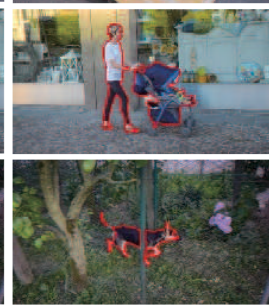

Ours

Fig. 6. Qualitative results and comparisons.

DAVIS: We also evaluated our method based on the entire DAVIS data set [27] in comparisons with some state-of-the-art supervised techniques, including the recent techniques based on CNN models. Table 3 summarizes the results obtained for a representative subset of the benchmark, where the IoU scores for the data set were computed over all 50 sequences. The records compared are all available from the DAVIS challenge website. ${ }^{1}$ The table shows that the overall performance of our method still remained competitive with non-CNN approaches, but inferior to that of CNN approaches. Based on these results, we must acknowledge the outstanding performance of an endto-end approach that needs to be pre-trained using a large-scale annotated data set.

Furthermore, we also find that the performance difference between "ours(1)" and "ours(5)" is not obvious in most cases. Thanks to the strong robustness of transductive learning, the classifier can be trained well enough with a small number of labeled samples. However, "ours(1)" clearly

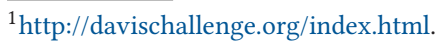


Table 1. Average Pixel Error (APE) Scores Based on the SegTrack Data Set (Where a Lower Score Is Better)

\begin{tabular}{lcccccccc}
\hline & supervised? & birdfall2 & cheetah & girl & monkeydog & parachute & penguin & average rank \\
\hline FST [25] & $\mathrm{N}$ & $217(5)$ & $890(5)$ & $3859(9)$ & $284(2)$ & $855(9)$ & - & 6.0 \\
POLP [5] & $\mathrm{N}$ & $278(9)$ & $824(3)$ & $1029(3)$ & $\mathbf{1 9 2 ( 1 )}$ & $251(7)$ & - & 4.6 \\
SAG [35] & $\mathrm{N}$ & $209(4)$ & $796(2)$ & $1040(4)$ & $562(9)$ & $\mathbf{2 0 7 ( 1 )}$ & - & 4.0 \\
FGS [19] & $\mathrm{N}$ & $242(7)$ & $1156(8)$ & $1564(7)$ & $483(7)$ & $328(8)$ & - & 7.4 \\
HVS [7] & $\mathrm{Y}$ & $305(10)$ & $1219(10)$ & $5777(10)$ & $493(8)$ & $1202(10)$ & 2116 & 9.6 \\
MCT [31] & $\mathrm{Y}$ & $252(8)$ & $1142(7)$ & $1304(6)$ & $563(10)$ & $235(6)$ & 1705 & 7.4 \\
SFP [10] & $\mathrm{Y}$ & $189(3)$ & $1170(9)$ & $2883(8)$ & $333(3)$ & $228(5)$ & 443 & 5.6 \\
TVS [34] & $\mathrm{Y}$ & $163(2)$ & $\mathbf{6 8 8 ( 1 )}$ & $1186(5)$ & $354(5)$ & $209(2)$ & 456 & 3.0 \\
Ours(1) & $\mathrm{Y}$ & $228(6)$ & $1076(6)$ & $1015(2)$ & $461(6)$ & $225(3)$ & 1030 & 4.6 \\
Ours(5) & $\mathrm{Y}$ & $\mathbf{1 5 9 ( 1 )}$ & $873(4)$ & $\mathbf{9 8 4 ( 1 )}$ & $342(4)$ & $225(3)$ & 893 & 2.6 \\
\hline
\end{tabular}

Additional APE rankings are also shown in parentheses.

Table 2. IoU Score Percentage on SegTrack.v2 Data Set (Higher Is Better)

\begin{tabular}{lccccccccc}
\hline & supervised? & soldier & frog & worm & monkey & bxm-person & drift-\#1 & hummingbird-\#1 & average \\
\hline FGS [19] & N & 83.8 & 72.3 & 82.8 & 84.8 & 85.4 & 74.8 & 54.4 & 76.9 \\
TS [37] & N & 76.3 & 80.2 & 82.4 & 83.1 & 86.1 & 70.7 & 53.0 & 75.9 \\
JOTS [36] & Y & 81.1 & 56.3 & 79.3 & 86.0 & $\mathbf{8 8 . 9}$ & 67.3 & 58.3 & 73.8 \\
OFL [32] & Y & $\mathbf{8 6 . 4}$ & 81.4 & $\mathbf{8 9 . 6}$ & $\mathbf{8 8 . 6}$ & 88.0 & $\mathbf{8 4 . 3}$ & 69.0 & $\mathbf{8 3 . 9}$ \\
Ours(1) & Y & 79.1 & 81.6 & 80.7 & 77.4 & 82.9 & 80.3 & 67.2 & 78.5 \\
Ours(5) & Y & 80.8 & $\mathbf{8 3 . 4}$ & 82.4 & 80.1 & 85.8 & 82.1 & $\mathbf{7 1 . 7}$ & 80.9 \\
\hline
\end{tabular}

Our method remained competitive with these methods, where OFL [32] benefited considerably from complicated iterative optimization.

falls behind "ours(5)" in some cases, e.g. "birdfall2," "cheetah," "monkeydog." This is due to the low quality and resolution of the input videos, which lead to mixed labeled samples that confuse the classifier. To this end, we suggest to initialize the algorithm with more than one labeled frame to train a valid model. Besides this, the other limitations of the proposed method are also discussed in Section 4.5. Anyway, we believe our method is very valuable from the theoretical point of view, though it is not that innovative to account for a new direction.

\subsection{Running Times}

All of the experiments were implemented in Matlab and run on a PC with a Dual-Core i5 CPU and 8 GB RAM. The computational cost depends on the video dimensions (width $\times$ height $\times$ length), so we tested the running times with only one example of an $854 \times 480 \times 50$ video sequence. The detailed performance for each frame is shown in Table 4. Overall, our method had a frame processing time of 4.70s. Due to the region-based segmentation and efficient optimization process, our method was faster than most of the other methods. They are computationally expensive, thus limiting their practical use (e.g., OFL [32] need almost one minute to process each frame, and CNN methods require quite a long time of GPU-powered online training for each input video).

\subsection{Limitation}

The main limitation of our method based on the utilization of long-range connections is the need to operate on larger image regions to ensure acceptable speed and memory usage, but this reduces the ability to handle fine image details. In particular, when the object is absorbed into the inseparable 
Table 3. IoU Score Percentage on a Representative Subset of the DAVIS Dataset (Higher is Better)

\begin{tabular}{lccc|ccccc}
\hline \multicolumn{3}{c}{ Recent CNN methods } & \multicolumn{5}{c}{ Recent non-CNN methods } \\
\hline Bear & MSK [26] & CTN [12] & VPN [11] & BVS [21] & FCP [28] & OFL [32] & Ours(1) & Ours(5) \\
Bmx-Bumps & 93.1 & 91.6 & 93.4 & $\mathbf{9 5 . 5}$ & 90.6 & 94.5 & 93.3 & 94.2 \\
Boat & $\mathbf{5 7 . 1}$ & 27.1 & 41.8 & 43.4 & 30.0 & 47.5 & 39.5 & 43.3 \\
Breakdance & 54.8 & 57.7 & 68.7 & 64.4 & 61.3 & $\mathbf{8 0 . 8}$ & 78.1 & 78.9 \\
Bus & $\mathbf{7 6 . 2}$ & 63.9 & 46.5 & 50.0 & 56.7 & 75.6 & 56.8 & 58.2 \\
Car-Turn & $\mathbf{8 9 . 1}$ & 74.3 & 86.3 & 85.3 & 83.2 & 67.7 & 80.3 & 81.4 \\
Dance-Twirl & 88.6 & 91.2 & $\mathbf{9 3 . 1}$ & 84.4 & 72.4 & 87.6 & 83.0 & 83.5 \\
Flamingo & $\mathbf{8 4 . 4}$ & 76.9 & 62.8 & 49.2 & 47.1 & 53.9 & 48.7 & 51.9 \\
Kite-Surf & 79.0 & 59.6 & 52.7 & $\mathbf{8 8 . 1}$ & 71.7 & 88.5 & 85.4 & 86.0 \\
Libby & 60.0 & $\mathbf{6 4 . 6}$ & 62.3 & 42.5 & 57.7 & 70.3 & 45.2 & 48.7 \\
Mallard-Fly & 77.5 & 73.0 & 72.6 & 77.6 & 31.6 & 55.3 & 76.3 & $\mathbf{7 8 . 3}$ \\
Motocross-Jump & 57.3 & 67.5 & $\mathbf{7 9 . 7}$ & 60.6 & 54.1 & 55.1 & 59.7 & 62.6 \\
Motorbike & $\mathbf{6 8 . 5}$ & 56.9 & $\mathbf{7 2 . 8}$ & 34.1 & 51.1 & 60.7 & 58.2 & 60.1 \\
Parkour & 56.6 & $\mathbf{8 1 . 3}$ & 80.8 & 56.3 & 71.3 & 47.6 & 72.0 & 74.9 \\
Scooter-Black & $\mathbf{8 8 . 2}$ & 85.0 & 87.3 & 75.6 & 32.2 & 85.9 & 74.9 & 75.8 \\
Soapbox & $\mathbf{8 2 . 5}$ & 61.1 & 60.5 & 33.7 & 50.4 & 80.7 & 66.9 & 68.2 \\
Soccerball & $\mathbf{8 9 . 9}$ & 83.0 & 81.1 & 78.9 & 44.9 & 68.7 & 77.4 & 78.1 \\
Swing & $\mathbf{8 9 . 0}$ & 81.8 & 77.2 & 84.4 & 82.0 & 7.9 & 75.6 & 77.7 \\
Stroller & 81.9 & 82.1 & $\mathbf{8 2 . 5}$ & 78.4 & 64.8 & 56.2 & 72.9 & 74.0 \\
Train & $\mathbf{8 5 . 4}$ & 76.8 & 84.2 & 76.7 & 59.7 & 88.3 & 77.2 & 77.9 \\
Dataset IoU & 90.4 & 86.4 & 78.0 & 87.2 & 84.1 & 91.2 & 90.8 & $\mathbf{9 1 . 4}$ \\
\hline D0.3 & 75.5 & 75.0 & 66.5 & 63.1 & 71.1 & 67.2 & 69.3 \\
\hline
\end{tabular}

Dataset IoU is computed over all 50 videos. Though our method is inferior to that of CNN approaches, it still remained competitive with non-CNN approaches, where OFL [32] requires much more computation costs.

Table 4. Detailed Running Times Based on an $854 \times 480 \times 50$ Video Sequence

\begin{tabular}{cc}
\hline Algorithm components & Running time (seconds per frame) \\
\hline adaptive over-segmentation & 0.76 \\
color histogram $f(x)$ & 0.31 \\
transductive learning & 1.27 \\
SIFT extraction & 1.13 \\
graph construction & 0.89 \\
graph-cut & 0.34 \\
\hline total & 4.70 \\
\hline
\end{tabular}

background, it is challenging to generate an accurate boundary in these low contrast regions by over-segmentation. Some typical examples are shown in Figures 7(a)-7(c), which demonstrate why our method struggles to compete with pixel-based methods [13, 21, 32]. The second limitation is that our high-order potential does not work for indistinctive regions. Thus, a region with few "words" (extracted SIFT point) has a low constraint with $E_{h}(X)$, as shown in Figures $7(\mathrm{~d})-7(\mathrm{f})$. Moreover, thin parts attached to the main body of the object are likely to be smoothed away by the MRF smoothing. 


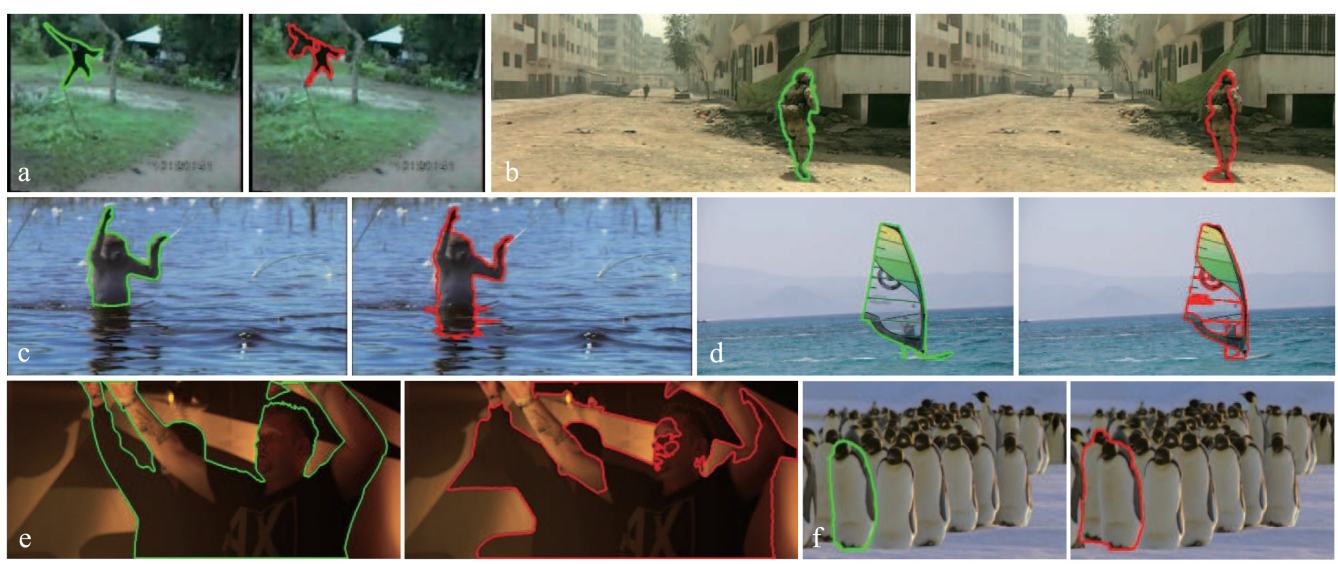

Fig. 7. Some typical segmentation errors. The green contour is the ground truth and the red contour is that segmented by our method.

\section{CONCLUSION}

In this study, we proposed an efficient and effective technique based on an MRF for unconstrained video object segmentation. Compared with other methods, an additional high-order potential is employed in this method. In particular, we compute the potential for each node using the multinomial event model, where the nodes with similar visual features are specified as soft preferences that need to be assigned the same label. This global clique is the key to our approach, because it ensures long-term appearance consistency during segmentation. Furthermore, we introduced an efficient approach for optimizing this intractable potential without requiring a complicated iterative process. Our qualitative and quantitative evaluation experiments demonstrated the effectiveness of the proposed method.

\section{REFERENCES}

[1] Sergi Caelles, Alberto Montes, Kevis-Kokitsi Maninis, Yuhua Chen, Luc Van Gool, Federico Perazzi, and Jordi PontTuset. 2018. The 2018 DAVIS challenge on video object segmentation. Retrieved from arXiv:1803.00557.

[2] Yadang Chen, Chuanyan Hao, Alex X. Liu, and Enhua Wu. 2019. Multi-level model for video object segmentation based on supervision optimization. IEEE Trans. Multimedia 99 (2019), 1-1. Retrieved from https://doi.org/10.1109/ TMM.2018.2890361.

[3] Yadang Chen, Chuanyan Hao, and Enhua Wu. 2018. Efficient frame-sequential label propagation for video object segmentation. Multimedia Tools Appl. 77, 5 (2018), 6117-6133.

[4] A. Faktor and M. Irani.2014. Video segmentation by non-local consensus voting. In Proceedings of the British Machine Vision Conference.

[5] Daniela Giordano, Francesca Murabito, Simone Palazzo, and Concetto Spampinato. 2015. Superpixel-based video object segmentation using perceptual organization and location prior. In Proceedings of the IEEE Conference on Computer Vision and Pattern Recognition (CVPR'15). 4814-4822.

[6] Ross Girshick, Jeff Donahue, Trevor Darrell, and Jitendra Malik. 2014. Rich feature hierarchies for accurate object detection and semantic segmentation. In Proceedings of the IEEE Conference on Computer Vision and Pattern Recognition (CVPR'14).

[7] Matthias Grundmann, Vivek Kwatra, Mei Han, and Irfan Essa. 2010. Efficient hierarchical graph-based video segmentation. In Proceedings of the IEEE Conference on Computer Vision and Pattern Recognition.

[8] K. He, X. Zhang, S. Ren, and J. Sun. 2016. Deep residual learning for image recognition. In Proceedings of the IEEE Conference on Computer Vision and Pattern Recognition (CVPR'16). 770-778.

[9] Fairouz Hussein and Massimo Piccardi. 2017. V-JAUNE: A framework for joint action recognition and video summarization. ACM Trans. Multimedia Comput. Commun. Appl. 13, 2, Article 20 (Apr. 2017). DOI : https://doi.org/10.1145/ 3063532 
[10] Suyog Dutt Jain and Kristen Grauman. 2014. Supervoxel-consistent foreground propagation in video. In Proceedings of the European Conference on Computer Vision: Part IV (Lecture Notes in Computer Science). Springer, 656-671.

[11] V. Jampani, R. Gadde, and P. V. Gehler. 2017. Video propagation networks. In Proceedings of the IEEE Conference on Computer Vision and Pattern Recognition.

[12] W. D. Jang and C. S. Kim. 2017. Online video object segmentation via convolutional trident network. In Proceedings of the IEEE Conference on Computer Vision and Pattern Recognition.

[13] Won-Dong Jang, Chulwoo Lee, and Chang-Su Kim. 2016. Primary object segmentation in videos via alternate convex optimization of foreground and background distributions. In Proceedings of the IEEE Conference on Computer Vision and Pattern Recognition.

[14] M. Keuper, B. Andres, and T. Brox. 2015. Motion trajectory segmentation via minimum cost multicuts. In Proceedings of the IEEE International Conference on Computer Vision.

[15] Johannes Kiess, Stephan Kopf, Benjamin Guthier, and Wolfgang Effelsberg. 2018. A survey on content-aware image and video retargeting. ACM Trans. Multimedia Comput. Commun. Appl. 14, 3, Article 76 (July 2018). DOI : https://doi org $/ 10.1145 / 3231598$

[16] Yeong Jun Koh and Chang-Su Kim. 2017. Primary object segmentation in videos based on region augmentation and reduction. In Proceedings of the IEEE Conference on Computer Vision and Pattern Recognition.

[17] Pushmeet Kohli, L'Ubor Ladický, and Philip H. Torr. 2009. Robust higher order potentials for enforcing label consistency. Int. 7. Comput. Vision 82, 3 (May 2009), 302-324.

[18] Alex Krizhevsky, Ilya Sutskever, and Geoffrey E. Hinton. 2012. ImageNet classification with deep convolutional neural networks. In Proceedings of the 25th International Conference on Neural Information Processing Systems. Curran Associates Inc., 1097-1105.

[19] Fuxin Li, Taeyoung Kim, Ahmad Humayun, David Tsai, and James M. Rehg. 2013. Video segmentation by tracking many figure-ground segments. Proceedings of the IEEE International Conference on Computer Vision. 2192-2199.

[20] Fei-Fei Li and Pietro Perona. 2005. A Bayesian hierarchical model for learning natural scene categories. In Proceedings of the IEEE Computer Society Conference on Computer Vision and Pattern Recognition (CVPR'05). IEEE Computer Society, Washington, DC, 524-531.

[21] Nicolas Maerki, Federico Perazzi, Oliver Wang, and Alexander Sorkine-Hornung. 2016. Bilateral space video segmentation. In Proceedings of the IEEE Conference on Computer Vision and Pattern Recognition.

[22] Andrew McCallum and Kamal Nigam. 1998. A comparison of event models for Naive Bayes text classification. In Proceedings of the AAAI Workshop on Learning for Text Categorization.

[23] Peter Ochs, Jitendra Malik, and Thomas Brox. 2014. Segmentation of moving objects by long-term video analysis. IEEE Trans. Pattern Anal. Mach. Intell. 36, 6 (2014), 1187-1200.

[24] Zhaoqing Pan, Jianjun Lei, Yajuan Zhang, and Fu Lee Wang. 2018. Adaptive fractional-pixel motion estimation skipped algorithm for efficient HEVC motion estimation. ACM Trans. Multimedia Comput. Commun. Appl. 14, 1, Article 12 (Jan. 2018). DOI : https://doi.org/10.1145/3159170

[25] Anestis Papazoglou and Vittorio Ferrari. 2013. Fast object segmentation in unconstrained video. In Proceedings of the IEEE International Conference on Computer Vision.

[26] F. Perazzi, A. Khoreva, R. Benenson, B. Schiele, and A. Sorkine-Hornung. 2017. Learning video object segmentation from static images. In Proceedings of the IEEE Conference on Computer Vision and Pattern Recognition.

[27] F. Perazzi, J. Pont-Tuset, B. McWilliams, L. Van Gool, M. Gross, and A. Sorkine-Hornung. 2016. A benchmark dataset and evaluation methodology for video object segmentation. In Proceedings of the IEEE Conference on Computer Vision and Pattern Recognition.

[28] Federico Perazzi, Oliver Wang, Markus Gross, and Alexander Sorkine-Hornung. 2015. Fully connected object proposals for video segmentation. In Proceedings of the IEEE International Conference on Computer Vision. 3227-3234.

[29] Jordi Pont-Tuset, Federico Perazzi, Sergi Caelles, Pablo Arbeláez, Alexander Sorkine-Hornung, and Luc Van Gool. 2017. The 2017 DAVIS challenge on video object segmentation. Retrieved from arXiv:1704.00675.

[30] Karen Simonyan and Andrew Zisserman. 2015. Very deep convolutional networks for large-scale image recognition. (Apr. 2015). Retrieved from arXiv:1409.1556v6.

[31] David Tsai, Matthew Flagg, Atsushi Nakazawa, and James M. Rehg. 2012. Motion coherent tracking using multi-label MRF optimization. Int. J. Comput. Vision 100, 2 (Nov. 2012), 190-202.

[32] Yi-Hsuan Tsai, Ming-Hsuan Yang, and Michael J. Black. 2016. Video segmentation via object flow. In Proceedings of the IEEE Conference on Computer Vision and Pattern Recognition.

[33] Lucas Pascotti Valem, Carlos Renan De Oliveira, Daniel Carlos Guimarães Pedronette, and Jurandy Almeida. 2018. Unsupervised similarity learning through rank correlation and kNN sets. ACM Trans. Multimedia Comput. Commun. Appl. 14, 4, Article 80 (Oct. 2018). DOI : https://doi.org/10.1145/3241053

[34] Botao Wang, Zhihui Fu, Hongkai Xiong, and Yuan Zheng. 2017. Transductive video segmentation on tree-structured model. IEEE Trans. Circ. Syst. Video Technol. 27, 5 (2017), 992-1005. 
[35] Wenguan Wang, Jianbing Shen, and Fatih Porikli. 2015. Saliency-aware geodesic video object segmentation. In Proceedings of the IEEE Conference on Computer Vision and Pattern Recognition. IEEE Computer Society, 3395-3402.

[36] Longyin Wen, Dawei Du, Zhen Lei, Stan Z. Li, and Ming-Hsuan Yang. 2015. JOTS: Joint online tracking and segmentation. In Proceedings of the Conference on Computer Vision and Pattern Recognition (CVPR'15). IEEE Computer Society, 2226-2234.

[37] Fanyi Xiao and Yong Jae Lee. 2016. Track and segment: An iterative unsupervised approach for video object proposals. In Proceedings of the IEEE Conference on Computer Vision and Pattern Recognition.

[38] Chenliang Xu and Jason J. Corso. 2016. LIBSVX: A supervoxel library and benchmark for early video processing. Int. f. Comput. Vision 119, 3 (Sept. 2016), 272-290.

[39] Zenglin Xu, Rong Jin, Jianke Zhu, Irwin King, and Michael R. Lyu. 2007. Efficient convex relaxation for transductive support vector machine. In Proceedings of the 20th International Conference on Neural Information Processing Systems (NIPS'07). Curran Associates, 1641-1648.

[40] Jiong Yang, Brian Price, Xiaohui Shen, Zhe Lin, and Junsong Yuan. 2016. Fast appearance modeling for automatic primary video object segmentation. IEEE Trans. Image Process. 25, 2 (2016), 503-515.

Received November 2018; revised January 2019; accepted February 2019 